\title{
Mobility, Emotion, and Universality in Future Collaboration
}

\author{
Mark Chignell $^{1}$, Naotsune Hosono ${ }^{2}$, Deborah Fels ${ }^{3}$, Danielle Lottridge ${ }^{1}$, \\ and John Waterworth ${ }^{4}$ \\ ${ }^{1}$ Dept. of Mechanical \& Industrial Engineering, University of Toronto \\ ${ }^{2}$ Oki Consulting Solutions, Tokyo \\ ${ }^{3}$ School of Information Technology Management, Ryerson University \\ ${ }^{4}$ Department of Informatics, Umea University
}

\begin{abstract}
The Graphical user interface has traditionally supported personal productivity, efficiency, and usability. With computer supported cooperative work, the focus has been on typical people, doing typical work in a highly rational model of interaction. Recent trends towards mobility, and emotional and universal design are extending the user interface paradigm beyond the routine. As computing moves into the hand and away from the desktop, there is a greater need for dealing with emotions and distractions. Busy and distracted people represent a new kind of disability, but one that will be increasingly prevalent. In this panel we examine the current state of the art, and prospects for future collaboration in non-normative computing requirements. This panel draws together researchers who are studying the problems of mobility, emotion and universality. The goal of the panel is to discuss how progress in these areas will change the nature of future collaboration.
\end{abstract}

Keywords: Future Interfaces; Mobile Computing; Perceptual Interfaces; Emotional Design; University Interfaces; Cross-Cultural Interfaces.

\section{Panel Description}

The future belongs to networked computing with multiple computing devices per person each being used in different ways and contexts. As devices become more flexible and more varied, there are increasing prospects for accommodating the needs of a wide range of people in many different contexts. In this panel we seek to explore the prospects for future online collaboration focusing on the issues of mobility, emotion, and universality. Our aim is to encourage debate concerning the common principles that emerge when we link non-normative forms of interaction into future collaborative interfaces.

The topics discussed in this panel are shown below.

- Mobile Devices (Chignell). Chignell will argue that mobile devices are likely to be the dominant interfaces for future collaboration. He will also discuss the disabilities implied by mobile interaction and the design principles that 
emerge in this context. In making this argument he will discuss a series of research projects carried out at the University of Toronto that have examined communication and collaboration within healthcare teams and between medical workers and patients using handheld devices.

- Perceptual Interfaces (Waterworth). Waterworth has a particular interest in the extent to which people feel present within a medium when interacting with or through a device. The ultimate in mediated presence has been thought of as total immersion in a virtual world - with the assumption that a virtual world is an alternative to the physical world, and in competition with it. This is no longer a viable view. In his view, the future won't be so much about interaction in either mediated or physical reality, but about being in unconstrained situations in which both are blended and presence levels are adjusted dynamically and automatically. For this to work, media devices will need to be sensitive to both the situational context of their use, and the state of their users.

- Emotional Design (Lottridge). Ms. Lottridge will discuss her Ph.d work at the University of Toronto on assessment of emotions during interaction as a means to bring the construct of emotional interface design into practical use. In addition to reviewing emotional assessment methods she will also review psychometric constructs such as emotional bandwidth, as a way to quantify the ability to experience and understand emotion. Varying emotional bandwidth capacities, and individual differences in emotional reporting, reveal disabilities or inequalities between people that should be measured in order to be accommodated. The broader implications for the use of emotion in interface design will also be discussed.

- Universal Interfaces (Fels). Dr. Fels will discuss how future interfaces can be designed to be more inclusive, and accommodating, with respect to the requirements of people with a range of abilities and disabilities. She will discuss the principles of universal design and how they can be applied in electronic media. Examples of inclusive media and sign language-based interfaces will be demonstrated.

- Universal and cross-cultural Interfaces (Hosono). The WWC (WorkWel Communicator) system has been developed by Dr. Hosono's group to address problems such as social isolation and reduced options for synchronous communication facing physically challenged persons in their efforts to fulfill their work duties. WWC is a multiple site voice system offering a realistic meeting experience for teleworkers with physical disabilities, while having a simplified interface that requires less effort to use (including simple conference control using a ten-key pad and voice out for dysphonea). This presentation will discuss how lessons learned in developing WWC can be applied to future collaboration systems for people with disabilities. 